\title{
The association between inflammatory potential of diet and disease activity: results from a cross-sectional study in patients with inflammatory bowel disease
}

\author{
Carlijn R. Lamers ${ }^{1,2^{*}}$ (D) Nicole M. de Roos $^{1}$ and Ben J. M. Witteman ${ }^{1,2}$
}

\begin{abstract}
Background: Diet may play a role in disease status in patients with inflammatory bowel disease. We tested whether the inflammatory potential of diet, based on a summation of pro- and anti-inflammatory nutrients, is associated with disease activity in patients with Crohn's disease and ulcerative colitis.

Methods: Participants completed a disease activity questionnaire (short Crohn's Disease Activity (sCDAl) or Patient Simple Clinical Colitis Activity Index (P-SCCAI)) and a Food Frequency Questionnaire (FFQ). FFQ data were used to calculate the Dietary Inflammatory Index (DII) which enables categorization of individuals' diets according to their inflammatory potential on a continuum from pro- to anti-inflammatory. Associations with disease activity were investigated by multiple linear regression.

Results: The analysis included 329 participants; 168 with Crohn's disease (median sCDAl score 93 [IQR 47-156]), and 161 with ulcerative colitis (median P-SCCAI score 1 [IQR 1-3]). Mean DII was $0.71 \pm 1.33$, suggesting a slightly proinflammatory diet. In Crohn's disease, the DII was positively associated with disease activity, even after adjustment for confounders $(p=0.008)$. The mean DII was significantly different between participants in remission and with mild and moderately active disease $(0.64,0.97$ and 1.52 respectively, $p=0.027)$. In ulcerative colitis, the association was not significant.

Conclusions: Disease activity was higher in IBD participants with a more pro-inflammatory diet with statistical significance in Crohn's disease. Although the direction of causality is not clear, this association strengthens the role for diet in medical treatment, which should be tested in an intervention study.
\end{abstract}

Keywords: Crohn's disease, Ulcerative colitis, Diet, Inflammation, Dietary inflammatory index

\footnotetext{
* Correspondence: carlijn.lamers@wur.nl

${ }^{1}$ Division of Human Nutrition and Health, Wageningen University \& Research (WUR), Stippeneng 4, 6708, WE, Wageningen, The Netherlands

${ }^{2}$ Department of Gastroenterology and Hepatology, Hospital Gelderse Vallei, Ede, The Netherlands
}

(c) The Author(s). 2020 Open Access This article is licensed under a Creative Commons Attribution 4.0 International License, which permits use, sharing, adaptation, distribution and reproduction in any medium or format, as long as you give appropriate credit to the original author(s) and the source, provide a link to the Creative Commons licence, and indicate if changes were made. The images or other third party material in this article are included in the article's Creative Commons licence, unless indicated otherwise in a credit line to the material. If material is not included in the article's Creative Commons licence and your intended use is not permitted by statutory regulation or exceeds the permitted use, you will need to obtain permission directly from the copyright holder. To view a copy of this licence, visit http://creativecommons.org/licenses/by/4.0/. The Creative Commons Public Domain Dedication waiver (http://creativecommons.org/publicdomain/zero/1.0/) applies to the data made available in this article, unless otherwise stated in a credit line to the data. 


\section{Background}

Dietary intake seems to play a role in the development of Crohn's disease (CD) and ulcerative colitis (UC), and possibly also in maintenance of remission and improvement of quality of life in patients with one of these inflammatory bowel diseases (IBD) [1, 2]. The exact mechanism is unknown, but modification of the gut microbiota and influence on immunological processes seem to be important $[3,4]$.

Diet is a modifiable lifestyle factor in which IBD patients seem to be interested and that may have beneficial effects on the course of IBD. Surveys performed on dietary beliefs and behaviour showed that around $60 \%$ of patients believe that diet influences their disease course and up to $77 \%$ of patients reported avoidance of particular foods to prevent or treat a flare [5-7]. There is cumulative evidence that certain components of a diet have anti- or pro-inflammatory properties and may therefore influence the course of disease $[3,8]$. This has led to the development of the Dietary Inflammatory Index (DII). With this DII, diets can be categorized from maximally anti-inflammatory to maximally pro-inflammatory. Assessment of the inflammatory potential of a diet takes into account that some foods contain both beneficial and unhealthy nutrients. Therefore, it is likely that the DII better reflects the influence of diet on inflammation and thus on the course of disease than analyses with single foods [911]. The DII has been used in several other patient groups to predict the dietary inflammatory potential related risk for development of disease or the influence on clinical course of disease [12-14].

Therefore, the aim of this study was to assess the association between the inflammatory potential of diet and disease activity in patients with inflammatory bowel disease, separated into CD and UC patients. We also investigated participants' self-perceived impact of diet on disease and whether they had made dietary modifications.

\section{Methods}

\section{Study design and study population}

In this cross-sectional study, IBD patients aged 18 years or older, diagnosed with either CD or UC, were included. Participants were recruited between July and October 2018. A total of 1035 patients received a personal invitation: 940 IBD patients of a local regional hospital in the Netherlands received a letter and 95 IBD patients from the Nijmegen Exercise Study - a longitudinal study to examine the impact of a physically active lifestyle on health, quality of life, development and progression of various (chronic) diseases - received an email with an invitation to participate. Furthermore, an unknown number of patients responded to an invitation via digital newsletters and the website of the Dutch IBD patient association. In total, 397 patients sent us an e-mail to show interest in the study and received access to our online questionnaire. Unfortunately, we did not register the source each participant originated from. Participation comprised an online questionnaire composed of questions regarding participants characteristics, a disease activity questionnaire (short Crohn's Disease Activity (sCDAI) or Patient Simple Clinical Colitis Activity Index (P-SCCAI)) and a Food Frequency Questionnaire (FFQ) [15-17]. It took participants about $40 \mathrm{~min}$ to complete the whole questionnaire. Participants were excluded in case of indeterminate colitis or unknown IBD type, missing or incomplete FFQ data or an implausible energy intake $(<800$ or $>4000 \mathrm{kcal}$ per day for men and $<500$ or $>3500 \mathrm{kcal}$ per day for women) to limit errors due to misreporting [18].

The medical ethical committee of Wageningen University decided that no formal ethical approval was needed, due to the low burden and risk of the study. All participants provided digital informed consent.

\section{Data collection \\ Participant characteristics}

Information on age, gender, height and weight, level of education, type of IBD, age at diagnosis, current medication and supplement use, previous IBD-related surgeries, food allergies and smoking was retrieved from the online questionnaire.

\section{Disease activity}

Disease activity was evaluated using the short Crohn's Disease Activity Index (sCDAI) for CD and the Patient Simple Clinical Colitis Activity Index (P-SCCAI) for UC $[15,16]$. Disease activity scores were used as a continuous outcome measure and classified using previously validated cut-off points: remission (sCDAI < 150 or PSCCAI $\leq 2$ ), mildly active disease (sCDAI 150-219 or PSCCAI 3-5), moderately active disease (sCDAI 220-450 or P-SCCAI 6-11) and severely active disease (sCDAI > 450 or P-SCCAI $\geq 12$ ) $[15,19]$.

\section{Dietary intake}

Dietary intake was assessed using a 179-item validated Food Frequency Questionnaire (FFQ) designed to assess the intake of the Dutch population by capturing the foods consumed during the previous month [17]. Macronutrient intake was calculated and the FFQ was used to calculate the inflammatory potential of their habitual diet by using the DII. The DII is an index consisting of 45 food parameters developed by reviewing and scoring scientific articles on diet and inflammatory markers to be able to determine the inflammatory potential of a diet $[9,10]$. A DII score above zero represents a pro-inflammatory diet and a DII score below zero represents an anti-inflammatory diet. Several papers 
described the development of inflammatory indices and calculation of the DII [9-11]. In short, DII scores of each food parameter were calculated by subtraction of the standard global mean of a representative world database from the amount of the food parameter eaten estimated from an FFQ and dividing this value by its standard deviation [10]. This $\mathrm{Z}$-value was converted to a centred percentile score to minimize the effect of skewing and to achieve a symmetrical distribution. This centred percentile score was multiplied by the food parameter specific inflammatory effect score. All food parameter DII scores were summed to create an overall DII score. When calculated from all 45 parameters, the DII could theoretically range from -8.87 (maximally antiinflammatory) to +7.98 (maximally pro-inflammatory) [20]. In our study, data on 28 of the 45 parameters were available for inclusion in the overall DII score, namely: energy, protein, carbohydrate, total fat, saturated fat, cholesterol, trans fat, mono- and polyunsaturated fat, n3 and $n-6$ fatty acids, fibre, thiamine, riboflavin, niacin, vitamins $\mathrm{A}, \mathrm{B} 6, \mathrm{~B} 12, \mathrm{C}, \mathrm{D}$ and $\mathrm{E}$, zinc, iron, magnesium, selenium, folic acid, beta-carotene and alcohol. The remaining 17 parameters, mainly flavonoids, herbs and spices, were not available because not all food parameters could be assessed reliably with the FFQ we used or were not available in the food composition database.

\section{Patient reported impact and modification of diet}

Information on participants' self-perceived impact of diet on disease and whether they had made dietary modifications since their diagnosis was retrieved from the online questionnaire.

\section{Statistical analysis}

Normally distributed data are presented as mean \pm standard deviation (SD), skewed data as median with interquartile range (IQR) and categorical data as frequencies with proportions. To compare baseline characteristics and DII between CD and UC and between disease activity groups, Chi-square tests were performed for categorical data, and independent samples t-test and one-way analysis of variance (ANOVA) (or KruskallWallis when not normally distributed) were performed for continuous variables. Post-hoc analyses for disease activity groups were performed using the Bonferroni multiple comparisons test. Multiple linear regression was used to determine associations between inflammatory potential of diet and disease activity. Results were reported as $\beta$-coefficients with $95 \%$ confidence intervals (CI). A $p$-value of $<0.05$ was considered statistically significant. Statistical analysis was carried out using IBM SPSS Statistics version 24.

\section{Results}

\section{Participants characteristics}

In total, 329 participants were included in the analysis (Fig. 1). Of these 329 participants, 168 participants (51\%) had CD and 161 participants (49\%) had UC. The majority of participants was female, well-educated and had experienced two or less flare-ups in the last year. The number of participants classified as having severely active disease was too small to analyse as a separate group (UC, $n=2$ ), so they were included in the moderately active disease group. About two-thirds of participants were in remission. Participants with $\mathrm{CD}$ were slightly younger at the time of this study and at diagnosis, they used more immunosuppressants and biologicals, and had more IBD-related surgeries than patients with UC. Supplement use was comparable in CD and UC (Table 1).

\section{Dietary intake and inflammatory potential}

The total energy intake and intakes of protein, carbohydrates and fat were not significantly different between $\mathrm{CD}$ and UC. In the total population, the DII ranged from -2.32 to 4.10 with a mean of $0.71 \pm 1.33$, suggesting a slightly pro-inflammatory diet. No significant differences were found between $\mathrm{CD}$ and UC $(0.79 \pm 1.37$ vs $0.62 \pm$ 1.28, $p=0.245$ ) (Table 2). Multiple linear regression showed a positive association for $\mathrm{CD}$ disease activity scores and the DII in the crude model $(\beta=12.96 ; p=$ 0.002 ), suggesting that $C D$ participants with a higher disease activity consumed a more pro-inflammatory diet. After adjustment for age, age at diagnosis, gender, BMI and education level, this association remained $(\beta=11.86$; $p=0.008)$. No significant association was found between UC disease activity scores and the DII $(p=0.307)$ (Table 3). Across CD and UC disease activity groups, no significant differences were found regarding total energy, protein, carbohydrates and fat intake. In $\mathrm{CD}$, the mean DII was significantly more pro-inflammatory when disease activity was higher $(p=0.027)$, with a significant difference between the remission and moderately active disease group after post-hoc analyses $(p=0.030)$ (Table 2/Fig. 2). The parameters that contributed to differences in the DII were alcohol, monoand polyunsaturated fatty acids, $n-3$ and n- 6 fatty acids, magnesium, selenium, vitamin $\mathrm{A}$, vitamin $\mathrm{D}$ and niacin, all having anti-inflammatory effect scores. Lower intakes of these parameters led to a less antiinflammatory, so a more pro-inflammatory diet, in participants with a higher disease activity.

\section{Patient reported impact and modification of diet}

Of the 329 participants, 216 participants (66\%) reported that diet had an impact on the course of their disease (73\% CD vs $58 \%$ UC; $p=0.007$ ). Since their diagnosis, 216 participants $(66 \%)$ have adjusted their dietary intake. 


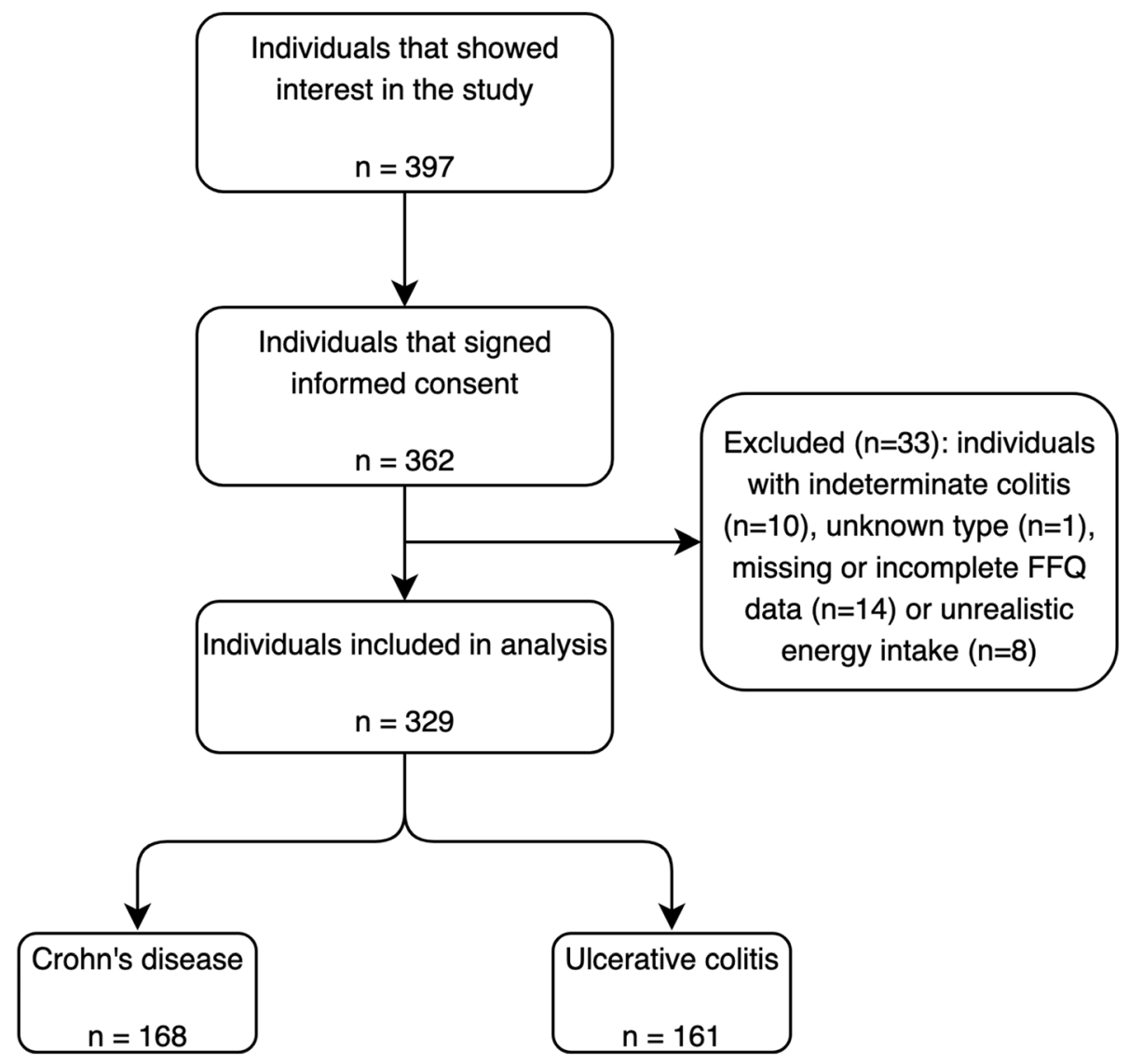

Fig. 1 Flowchart of individuals included in analysis

Generally, they reported avoidance of certain food products instead of a higher intake of beneficial foods. Lactose-containing products were mostly mentioned to be avoided (37\%) followed by spicy foods (23\%). Furthermore, participants reported that they reduced their intake of fat $(20 \%)$, meat (18\%), sugar (12\%) and onions (11\%). No differences were found between CD and UC or disease activity groups. Of the 329 participants, 194 participants (59\%) adjust their diet during a flare-up.

\section{Discussion}

In this study, disease activity was associated with the inflammatory potential of diet in participants with Crohn's disease. Participants with a more pro-inflammatory diet seem to have a higher disease activity. Whether this association is causal remains unclear. In participants with ulcerative colitis, the association was not significant. The majority of participants reported impact of diet on their disease. Modification of diet since diagnosis and during flare-ups was common in both patient groups and all disease activity groups.

To our knowledge, this is the first study to investigate the association between the inflammatory potential of diet and disease activity in patients with IBD in such a large sample. The DII has been calculated previously in a case control study including 62 UC patients and 124 controls to predict the risk of UC based on the inflammatory potential of diet. In that cross-sectional study, participants with higher DII scores seemed to have a higher risk of UC [21]. An analysis of three large prospective cohorts showed that dietary patterns with high inflammatory potential were associated with an increased risk of developing $\mathrm{CD}$, but not $\mathrm{UC}$, though they used a slightly different inflammatory index [2]. In contrast to our results, a recently published cross-sectional study in Iranian patients with an established IBD diagnosis did not find an association between the inflammatory potential of diet and disease activity [22]. The null finding in the Iranian patients might be due to the small sample size $(n=143)$, especially the small number of participants with $\mathrm{CD}(n=32)$, as compared to $178 \mathrm{CD}$ patients in the present study.

The DII consists of 45 food parameters that all have their own inflammatory effect score. The inflammatory effect scores we used varied from -0.663 for fibre to + 0.373 for saturated fat. The contribution of a food parameter to the total DII score depends on the individuals' intake and the deviation of the inflammatory effect score 
Table 1 Characteristics of the study population consisting of 168 CD and 161 UC participants

\begin{tabular}{|c|c|c|c|c|c|c|c|c|}
\hline & & & & ase activity & & & se activity $U$ & \\
\hline & $C D$ & $U C$ & Remission & Mild & Moderate & Remission & Mild & Moderate \\
\hline Subjects, n (\%) & $168(51)$ & $161(49)$ & $123(73)$ & $27(16)$ & $18(11)$ & $108(67)$ & $38(24)$ & $15(9)$ \\
\hline Gender, n (\%) & & & & & & & & \\
\hline Female & $117(70)$ & $97(60)$ & $84(68)$ & $17(63)$ & $16(89)$ & $60(56)$ & $27(71)$ & $10(67)$ \\
\hline Age (years) & $47 \pm 16^{*}$ & $51 \pm 15^{*}$ & $48 \pm 16$ & $46 \pm 17$ & $47 \pm 14$ & $50 \pm 14$ & $54 \pm 16$ & $45 \pm 16$ \\
\hline Age at diagnosis (years) & $32 \pm 15^{*}$ & $36 \pm 15^{*}$ & $33 \pm 16$ & $30 \pm 14$ & $33 \pm 9$ & $35 \pm 14$ & $41 \pm 17$ & $32 \pm 15$ \\
\hline BMI $\left(\mathrm{kg} / \mathrm{m}^{2}\right)$ & $24.8 \pm 4.7$ & $24.9 \pm 3.8$ & $24.8 \pm 4.4$ & $24.9 \pm 5.1$ & $25.0 \pm 6.6$ & $24.7 \pm 3.6$ & $25.4 \pm 3.9$ & $24.8 \pm 4.9$ \\
\hline Smoking, n (\%) & & & & & & & & \\
\hline Never & $123(73)$ & $124(77)$ & $97(79)^{\mathrm{a}}$ & $17(63)^{a, b}$ & $9(50)^{b}$ & $80(74)$ & $31(82)$ & $13(87)$ \\
\hline Current & $16(10)$ & $7(4)$ & $8(7)^{\mathrm{a}}$ & $6(22)^{b}$ & $2(11)^{a, b}$ & $5(5)$ & $1(3)$ & $1(7)$ \\
\hline Former & $29(17)$ & $30(19)$ & $18(15)^{\mathrm{a}}$ & $4(15)^{a, b}$ & $7(39)^{b}$ & $23(21)$ & $6(16)$ & $1(7)$ \\
\hline Education level ${ }^{c}, \mathrm{n}(\%)$ & & & & & & & & \\
\hline Low & $37(22)$ & $34(21)$ & $25(20)$ & $7(26)$ & $5(28)$ & $21(19)$ & $10(26)$ & $3(20)$ \\
\hline Middle & $52(31)$ & $48(30)$ & $38(31)$ & $5(19)$ & $9(50)$ & $33(31)$ & $8(21)$ & $7(47)$ \\
\hline High & $79(47)$ & $79(49)$ & $60(49)$ & $15(56)$ & $4(22)$ & $54(50)$ & $20(53)$ & $5(33)$ \\
\hline Medication use, n (\%) & & & & & & & & \\
\hline Mesalazines & $28(17)^{* *}$ & $97(60)^{* *}$ & $21(17)$ & $4(15)$ & $3(17)$ & $65(60)$ & $21(55)$ & $11(73)$ \\
\hline Corticosteroids & $22(13)$ & $24(15)$ & $13(11)$ & $6(22)$ & $3(17)$ & $9(8)^{\mathrm{a}}$ & $10(26)^{b}$ & $5(33)^{b}$ \\
\hline Immunosuppressants & $70(42)^{* *}$ & $33(21)^{* *}$ & $52(42)$ & $11(41)$ & $7(39)$ & $20(19)$ & $9(24)$ & $4(27)$ \\
\hline Biologicals & $58(35)^{* *}$ & $27(17)^{* *}$ & $36(29)$ & $12(44)$ & $10(56)$ & $11(10)^{a}$ & $9(24)^{a, b}$ & $7(47)^{b}$ \\
\hline Other & $24(14)^{*}$ & $11(7)^{*}$ & $14(11)$ & $6(22)$ & $4(22)$ & $4(4)^{a}$ & $4(11)^{a, b}$ & $3(20)^{b}$ \\
\hline No medication use & $34(20)$ & $30(19)$ & $29(24)$ & $4(15)$ & $1(6)$ & $24(22)$ & $6(16)$ & $0(0)$ \\
\hline Flare-ups in past year, $\mathrm{n}(\%)$ & & & & & & & & \\
\hline None & $92(55)^{*}$ & $61(38)^{*}$ & $80(65)^{\mathrm{a}}$ & $7(26)^{b}$ & $5(28)^{b}$ & $50(46)^{\mathrm{a}}$ & $10(26)^{a, b}$ & $1(7)^{b}$ \\
\hline $1-2$ flare-ups & $55(33)^{*}$ & $70(44)^{*}$ & $35(29)$ & $14(52)$ & $6(33)$ & $45(42)$ & $20(53)$ & $5(33)$ \\
\hline 3-4 flare-ups & $8(5)^{*}$ & $19(12)^{*}$ & $2(2)^{a}$ & $4(15)^{b}$ & $2(11)^{a, b}$ & $8(7)^{a}$ & $4(11)^{a}$ & $7(47)^{b}$ \\
\hline More than 4 flare-ups & $13(7)$ & $11(7)$ & $6(5)^{\mathrm{a}}$ & $2(7)^{a, b}$ & $5(28)^{b}$ & $5(5)$ & $4(11)$ & $2(13)$ \\
\hline Supplement use, n (\%) & $74(44)$ & $68(42)$ & $54(44)$ & $11(41)$ & $9(50)$ & $39(36)$ & $21(55)$ & $8(53)$ \\
\hline Surgery, n (\%) & $57(34)^{* *}$ & $13(8)^{* *}$ & $40(33)$ & $8(30)$ & $9(50)$ & $6(6)$ & $6(16)$ & $1(7)$ \\
\hline Intolerances/allergies, n (\%) & $62(37)$ & $44(27)$ & $40(33)$ & $13(48)$ & $9(50)$ & $23(21)^{a}$ & $16(42)^{b}$ & $5(33)^{a, b}$ \\
\hline
\end{tabular}

Data are presented as mean $\pm \mathrm{SD}$ for normally distributed data. Categorical data are presented as $\mathrm{n}(\%) .{ }^{*} p<0.05 * * 0<0.001$

Abbreviations: CD Crohn's disease, UC Ulcerative colitis, BMI Body mass index ${ }^{\mathrm{a}, \mathrm{b}}$ groups with the same superscript letters do not differ significantly after post-hoc analyses using the Bonferroni test $(p>0.05)$

${ }^{C}$ Education level: no education, primary or lower vocational education and lower general secondary education (low); secondary vocational education and higher general secondary education (middle); higher vocational education and university (high)

from 0 . Of the food parameters available in this study, fibre, vitamin A, B6, C, D, E, magnesium, zinc, polyunsaturated fatty acids and n-3 fatty acids had the largest anti-inflammatory impact. Total fat and saturated fat had the largest pro-inflammatory impact. These antiand pro-inflammatory effects are all based on general inflammation, but seem to be in line with IBD specific nutrient studies. The anti-inflammatory potential of fibre is in line with a prospective study in which they found that high consumption of dietary fibre reduces the risk of relapse among $C D$ patients [23]. By bacterial fermentation of dietary fibre, the production of short-chain fatty acids increases which has anti-inflammatory effects [24]. However, in both the abovementioned study and the DII, no distinction is made between the fermentability, solubility and viscosity of fibres, factors that influence the therapeutic effects of consumption [25]. Regarding the before mentioned vitamins and minerals, several reviews have described the effect of deficiencies on IBD [26-28]. For example, clinical disease activity increases and quality of life decreases significantly with lower levels of vitamin $\mathrm{D}$, and zinc deficiency was shown to be correlated with inflammation in IBD by increasing the number of proinflammatory cells [26-28]. Poly-unsaturated and 
Table 2 Disease activity and inflammatory potential of diet of CD and UC participants and stratified for disease activity

\begin{tabular}{|c|c|c|c|c|c|c|c|c|}
\hline & \multirow[b]{2}{*}{$C D$} & \multirow[b]{2}{*}{ UC } & \multicolumn{3}{|c|}{ Disease activity CD } & \multicolumn{3}{|c|}{ Disease activity UC } \\
\hline & & & Remission & Mild & Moderate & Remission & Mild & Moderate \\
\hline \multicolumn{9}{|l|}{ Disease activity } \\
\hline sCDAl score & 93 [47-156] & - & $79[44-103]^{a}$ & $171[165-191]^{b}$ & $269[233-326]^{c}$ & - & - & - \\
\hline Range & $44-357$ & - & $44-146$ & $150-218$ & $220-357$ & - & - & - \\
\hline P-SCCAl score & - & $1[1-3]$ & - & - & - & $1[1-1]^{a}$ & $3[3-4]^{b}$ & $7[6-9]^{c}$ \\
\hline Range & - & $0-13$ & - & - & - & $0-2$ & $3-5$ & $6-13$ \\
\hline \multicolumn{9}{|c|}{ Dietary intake } \\
\hline \multicolumn{9}{|l|}{ Nutrient intake } \\
\hline Energy (kcal) & $1912 \pm 618$ & $2011 \pm 565$ & $1958 \pm 601$ & $1789 \pm 602$ & $1780 \pm 738$ & $2050 \pm 587$ & $1919 \pm 496$ & $1958 \pm 577$ \\
\hline Protein, EN\% & $15.0 \pm 2.5$ & $15.3 \pm 2.7$ & $15.1 \pm 2.4$ & $15.0 \pm 2.2$ & $14.6 \pm 3.1$ & $15.2 \pm 2.8$ & $15.4 \pm 2.6$ & $15.2 \pm 2.6$ \\
\hline Carbohydrates, EN\% & $43.4 \pm 7.3$ & $42.5 \pm 6.4$ & $42.9 \pm 7.4$ & $45.6 \pm 6.6$ & $43.9 \pm 7.2$ & $42.2 \pm 6.8$ & $43.1 \pm 5.7$ & $43.1 \pm 4.6$ \\
\hline Fat, EN\% & $36.4 \pm 6.2$ & $36.5 \pm 5.5$ & $36.7 \pm 6.1$ & $34.4 \pm 6.1$ & $37.3 \pm 6.8$ & $36.6 \pm 5.8$ & $36.1 \pm 5.2$ & $37.3 \pm 4.4$ \\
\hline DII & $0.79 \pm 1.37$ & $0.62 \pm 1.28$ & $0.64 \pm 1.29^{\mathrm{a}}$ & $0.97 \pm 1.51^{\mathrm{a}, \mathrm{b}}$ & $1.52 \pm 1.42^{b}$ & $0.51 \pm 1.28$ & $0.78 \pm 1.13$ & $0.98 \pm 1.61$ \\
\hline Range & $-2.22-3.99$ & $-2.32-4.10$ & $-2.22-3.94$ & $-1.53-3.61$ & $-1.06-3.99$ & $-2.32-3.46$ & $-1.04-2.64$ & $-0.86-4.10$ \\
\hline
\end{tabular}

Data are presented as mean \pm SD for normally distributed data or median [interquartile range] when skewed. Categorical data are presented as $\mathrm{n}$ (\%)

Abbreviations: CD Crohn's disease, UC Ulcerative colitis, sCDAI Short Crohn's Disease Activity Index, P-SCCAI Patient Simple Clinical Colitis Activity Index, EN\% Energy percent, DII Dietary inflammatory index

a, bgroups with the same superscript letters do not differ significantly after post-hoc analyses using the Bonferroni test $(p>0.05)$

especially n-3 fatty acids have mostly been investigated in the context of supplementation with controversial results [29]. Beneficial effects have been shown, although a clear protective effect in preventing clinical relapse is not demonstrated [29]. A prospective study in patients with $\mathrm{CD}$ showed that a diet higher in total fat, saturated fat and a higher ratio of n-6:n-3 fatty acids was associated with disease relapses, which is in line with the inflammatory effect scores of the DII [30]. It seems reasonable to increase dietary intake of $n-3$ fatty acids for anti-inflammatory effects taking into account the involvement of $\mathrm{n}-3$ fatty acids in immunological and inflammatory responses and an imbalance in n-6:n-3 fatty acid ratio to be a powerful pro-inflammatory stimulus [31]. All before mentioned studies combined, it is likely that the DII reflects influence of diet not only on general inflammation, but also on inflammation in IBD.

The differences in associations of inflammatory potential of diet with disease activity between $C D$ and $\mathrm{UC}$ found in this study, are in line with previous studies. Regarding dietary intake, trends are observed in $\mathrm{CD}$ as well as in UC, but significant effects are more commonly found in CD [32]. Although we had to use a different disease activity questionnaire for each type of IBD, there is a similar distribution of disease activity groups in $\mathrm{CD}$ and UC. This distribution makes it unlikely that the different questionnaires account for the differences in associations between $\mathrm{CD}$ and UC. We did not assess the affected part of the gastrointestinal tract. Therefore, it is not possible to correct the associations for this aspect, although the affected part of the gastrointestinal tract may explain differences in associations between CD and UC.

Dietary beliefs of the participants in our study are in line with previous surveys. A similar percentage (58$62 \%)$ reported that diet influences their disease course and that avoidance of certain food products is preferred over a higher intake of beneficial foods [5-7].

Strengths of this study include the large number of participants, which enabled us to perform analyses for

Table 3 Results of multiple linear regression of the association between DII and disease activity as continuous variables, for CD and UC

\begin{tabular}{|c|c|c|c|c|c|}
\hline & & \multicolumn{2}{|l|}{$C D(\boldsymbol{n}=168)$} & \multicolumn{2}{|l|}{ UC $(\boldsymbol{n}=161)$} \\
\hline & & $\beta$-coefficient (95\% Cl) & $\boldsymbol{p}$-value & $\beta$-coefficient (95\% Cl) & $\boldsymbol{p}$-value \\
\hline \multirow[t]{2}{*}{ DII } & Crude & $12.96(4.74-21.18)$ & 0.002 & $0.145(-0.134-0.424)$ & 0.307 \\
\hline & Adjusted* & $11.86(3.14-20.58)$ & 0.008 & $0.062(-0.236-0.361)$ & 0.681 \\
\hline
\end{tabular}

Abbreviations: CD Crohn's disease, UC Ulcerative colitis, DII Dietary inflammatory index, CI Confidence interval

$C D$ disease activity scores can range from 0 to $>450$ and UC disease activity scores can range from 0 to 19

Bold values are significant

${ }^{*}$ Adjusted model: adjusted for age (years), age at diagnosis (years), gender (m/f), BMI $\left(\mathrm{kg} / \mathrm{m}^{2}\right)$ and education level (low/middle/high) 


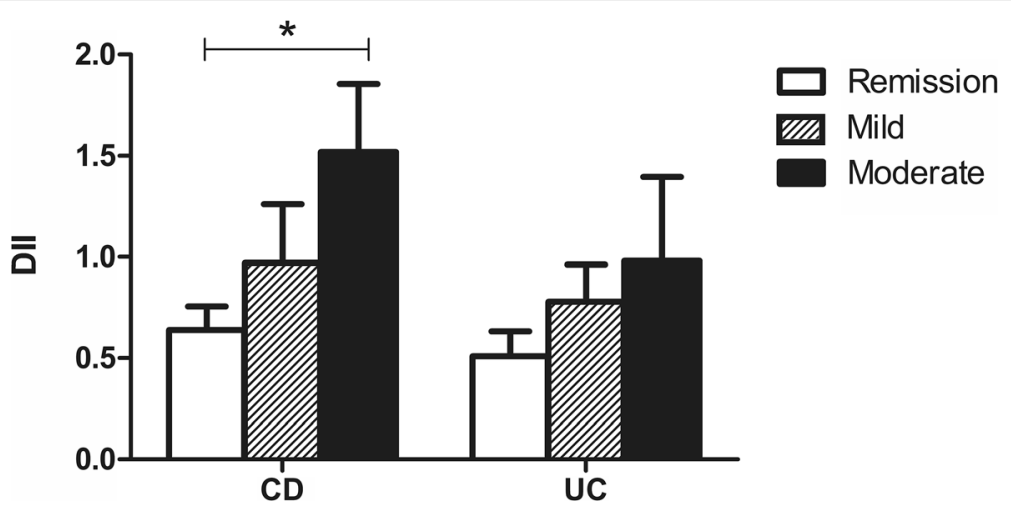

Fig. 2 Mean DII of CD $(n=168)$ and UC $(n=161)$ participants stratified for disease activity

Abbreviations: CD: Crohn's disease, UC: ulcerative colitis, Dll: dietary inflammatory index. Error bars represent standard error. ${ }^{*}$ indicates significant difference between groups $(p=0.03)$

$\mathrm{CD}$ and UC separately, and the use of validated questionnaires to determine disease activity and dietary intake. However, some limitations should be mentioned. This was a cross-sectional study and any association could therefore be a result of reverse causality. Participants who experience more pain or discomfort because of their disease may have changed their diet to relieve symptoms. Besides that, our sample included mainly participants in remission. A sample with an equal number of participants in each disease activity category would have increased the power to find stronger associations. Next to this, some extent of bias possibly occurred. More women than men responded, and the education level in our sample was high. In a large German study that compared responders to non-responders, women (up to 50 years old) were more likely to respond than men, as were non-smokers and those with a high education level [33]. As diet quality is related to gender, education level, and various other lifestyle factors [34], this could mean that on average, our study sample had a more healthy diet than that of the average IBD patient, corresponding with a lower DII (more antiinflammatory). Although, the range in DII was wide enough to find associations with disease activity, putting more effort into recruiting patients with more active disease or an unhealthy diet would be recommendable for the future. However, we do not think that selection bias negatively affected our study results, Another limitation is that we could not perform the complete calculation of the DII, because not all food parameters could be assessed with the FFQ or were not available in the food composition database. Those food parameters, mainly flavonoids, herbs and spices, all had anti-inflammatory effect scores. Therefore, our results were probably more directed towards pro-inflammatory scores. Using less food parameters generally also results in a lower variation in DII [11]. However, all other studies that calculated the DII also were unable to include all 45 food parameters and used a number of food parameters that was comparable to our study [12-14, 20, 21]. The DII only consists of food parameters, supplement use is not included. In our study, more than $40 \%$ of the participants used a food supplement, including vitamin supplements. Most vitamins and minerals do have antiinflammatory effect scores, which might have led to an underestimation of the DII in our study. As we did not have information about brands and dosages of the supplements, it was not possible to incorporate these in the calculation of the DII. However, supplement use was equal in $\mathrm{CD}$ and $\mathrm{UC}$ participants as well as all disease activity groups, which makes it less likely that supplement use affected the association between DII and disease activity. Finally, for the outcome disease activity, we did not use an objective marker, but based it on a questionnaire, which was filled in by the participant instead of a physician. However, previous studies have validated the SCDAI and P-SCCAI and concluded that both are reliable and feasible for disease activity measurement. For both participant-based disease activity questionnaires, significant correlations were found with the physician-based questionnaires as well as biomarkers such as CRP $[15,16]$.

\section{Conclusions}

In conclusion, we found an association between the inflammatory potential of diet and disease activity in Crohn's disease, but not in ulcerative colitis. Although this association does not prove a causal relationship, for daily practice it suggests that a diet high in antiinflammatory nutrients such as fibre, $\mathrm{n}-3$ fatty acids, vitamins and minerals, which is a diet in line with current nutritional guidelines for healthy adults, seems to be equally prudent in IBD patients, especially Crohn's disease. Longitudinal studies are needed to further investigate the effect of diet on the course of disease. 


\section{Abbreviations}

ANOVA: Analysis of variance; BMl: Body mass index; CD: Crohn's disease; Cl: Confidence interval; DII: Dietary Inflammatory Index; EN\%: Energy percent; FFQ: Food Frequency Questionnaire; IBD: Inflammatory bowel disease; IQR: Interquartile range; P-SCCAl: Patient Simple Clinical Colitis Activity Index; SCDAl: Short Crohn's Disease Activity Index; SD: Standard deviation; UC: Ulcerative colitis

\section{Acknowledgements}

We would like to thank all the participants that filled in our survey and the Crohn en Colitis Ulcerosa Vereniging (CCUVN) and Nijmegen Exercise Study for their support in participant recruitment.

\section{Authors' contributions}

C.L.: study concept and design, data acquisition, data analysis and interpretation, drafting manuscript. N.R.: study concept and design, critical revision of manuscript. B.W.: study concept and design, critical revision of manuscript. All authors have read and approved the manuscript.

\section{Funding}

This work was supported by a grant from the Province of Gelderland as part of the EAT2MOVE project (proposal PS2014-49). This grant was used to pay the salary of the corresponding author and the use of the FFQ.

\section{Availability of data and materials}

All data generated and/or analysed during the current study are available from the corresponding author on reasonable request.

\section{Ethics approval and consent to participate}

The medical ethical committee of Wageningen University decided that no formal ethical approval was needed, due to the low burden and risk of the study. All participants provided digital informed consent.

\section{Consent for publication}

Not applicable.

\section{Competing interests}

The authors declare that they have no competing interests.

Received: 30 April 2020 Accepted: 24 August 2020

Published online: 29 September 2020

\section{References}

1. Charlebois A, Rosenfeld G, Bressler B. The impact of dietary interventions on the symptoms of inflammatory bowel disease: a systematic review. Crit Rev Food Sci Nutr. 2016;56(8):1370-8.

2. Lo CH, Lochhead $\mathrm{P}$, Khalili $\mathrm{H}$, et al. Dietary inflammatory potential and risk of Crohn's disease and ulcerative colitis. Gastroenterology. 2020;500165085(20):30603.

3. Lee $\mathrm{D}$, Albenberg $\mathrm{L}$, Compher $\mathrm{C}$, et al. Diet in the pathogenesis and treatment of inflammatory bowel diseases. Gastroenterology. 2015;148(6): 1087-106.

4. Llewellyn SR, Britton GJ, Contijoch EJ, et al. Interactions between diet and the intestinal microbiota Alter intestinal permeability and colitis severity in mice. Gastroenterology. 2018;154(4):1037-46.e2.

5. Zallot C, Quilliot D, Chevaux JB, et al. Dietary beliefs and behavior among inflammatory bowel disease patients. Inflamm Bowel Dis. 2013;19(1):66-72.

6. Limdi JK, Aggarwal D, McLaughlin JT. Dietary practices and beliefs in patients with inflammatory bowel disease. Inflamm Bowel Dis. 2016;22(1): 164-70.

7. de Vries JHM, Dijkhuizen M, Tap P, Witteman BJM. Patient's dietary beliefs and Behaviours in inflammatory bowel disease. Dig Dis. 2019:37(2):131-9.

8. Tilg H, Moschen AR. Food, immunity, and the microbiome. Gastroenterology. 2015;148(6):1107-19.

9. Cavicchia PP, Steck SE, Hurley TG, et al. A new dietary inflammatory index predicts interval changes in serum high-sensitivity C-reactive protein. J Nutr. 2009:139(12):2365-72.

10. Shivappa N, Steck SE, Hurley TG, et al. Designing and developing a literature-derived, population-based dietary inflammatory index. Public Health Nutr. 2014;17(8):1689-96.
11. Hébert JR, Shivappa N, Wirth MD, et al. Perspective: the dietary inflammatory index (DII)-lessons learned, improvements made, and future directions. Adv Nutr. 2019;10(2):185-95.

12. Shivappa N, Godos J, Hebert JR, et al. Dietary inflammatory index and colorectal cancer risk-a meta-analysis. Nutrients. 2017;9:9.

13. Boden S, Wennberg M, Van Guelpen B, et al. Dietary inflammatory index and risk of first myocardial infarction; a prospective population-based study. Nutr J. 2017;16(1):21

14. da Costa Silva BY, de Carvalho Sampaio HA, Shivappa N, et al. Dietary inflammatory index and clinical course of multiple sclerosis. Eur J Clin Nutr. 2019:73(7):979-88.

15. Thia K, Faubion WA Jr, Loftus EV Jr, et al. Short CDAl: development and validation of a shortened and simplified Crohn's disease activity index. Inflamm Bowel Dis. 2011;17(1):105-11.

16. Bennebroek Evertsz F, Nieuwkerk PT, Stokkers PC, et al. The patient simple clinical colitis activity index (P-SCCAI) can detect ulcerative colitis (UC) disease activity in remission: a comparison of the P-SCCAI with clinicianbased SCCAI and biological markers. J Crohns Colitis. 2013;7(11):890-900.

17. Streppel MT, de Vries JH, Meijboom S, et al. Relative validity of the food frequency questionnaire used to assess dietary intake in the Leiden longevity study. Nutr J. 2013;12:75.

18. Willett W. Nutritional epidemiology. Oxford. New York: Oxford University Press; 2013

19. Walsh AJ, Ghosh A, Brain AO, et al. Comparing disease activity indices in ulcerative colitis. J Crohns Colitis. 2014;8(4):318-25.

20. Shivappa N, Hebert JR, Kivimaki M, Akbaraly T. Alternative healthy eating index 2010, dietary inflammatory index and risk of mortality: results from the Whitehall II cohort study and meta-analysis of previous dietary inflammatory index and mortality studies. Br J Nutr. 2017;118(3):210-21.

21. Shivappa N, Hébert JR, Rashvand S, et al. Inflammatory potential of diet and risk of ulcerative colitis in a case-control study from Iran. Nutr Cancer. 2016; 68(3):404-9.

22. Mirmiran P, Moslehi N, Morshedzadeh N, et al. Does the inflammatory potential of diet affect disease activity in patients with inflammatory bowel disease? Nutr J. 2019;18(1):65.

23. Brotherton CS, Martin CA, Long MD, et al. Avoidance of fiber is associated with greater risk of Crohn's disease flare in a 6-month period. Clin Gastroenterol Hepatol. 2016;14(8):1130-6.

24. Wong C, Harris PJ, Ferguson LR. Potential benefits of dietary fibre intervention in inflammatory bowel disease. Int J Mol Sci. 2016;17:6.

25. Holscher HD. Dietary fiber and prebiotics and the gastrointestinal microbiota. Gut Microbes. 2017:8(2):172-84.

26. Weisshof $\mathrm{R}$, Chermesh I. Micronutrient deficiencies in inflammatory bowel disease. Curr Opin Clin Nutr Metab Care. 2015;18(6):576-81.

27. Reich KM, Fedorak RN, Madsen K, Kroeker Kl. Vitamin D improves inflammatory bowel disease outcomes: basic science and clinical review. World J Gastroenterol. 2014;20(17):4934-47.

28. Rossi RE, Whyand T, Murray CD, et al. The role of dietary supplements in inflammatory bowel disease: a systematic review. Eur J Gastroenterol Hepatol. 2016;28(12):1357-64.

29. Scaioli E, Liverani E, Belluzzi A. The imbalance between $n-6 / n-3$ polyunsaturated fatty acids and inflammatory bowel disease: a comprehensive review and future therapeutic perspectives. Int J Mol Sci. 2017;18:12.

30. Tanaka M, Iwao $Y$, Sasaki $S$, et al. Moderate dietary temperance effectively prevents relapse of Crohn disease: a prospective study of patients in remission. Gastroenterol Nurs. 2007:30(3):202-10.

31. Cabré E, Mañosa M, Gassull MA. Omega-3 fatty acids and inflammatory bowel diseases - a systematic review. Br J Nutr. 2012;107(Suppl 2):S240-52.

32. Richman E, Rhodes JM. Review article: evidence-based dietary advice for patients with inflammatory bowel disease. Aliment Pharmacol Ther. 2013: 38(10):1156-71.

33. Enzenbach C, Wicklein B, Wirkner K, Loeffler M. Evaluating selection bias in a population-based cohort study with low baseline participation: the LIFEadult-study. BMC Med Res Methodol. 2019;19(1):135

34. Looman M, Feskens EJ, de Rijk M, et al. Development and evaluation of the Dutch healthy diet index 2015. Public Health Nutr. 2017;20(13):2289-99.

\section{Publisher's Note}

Springer Nature remains neutral with regard to jurisdictional claims in published maps and institutional affiliations. 